\title{
Cosmological singlet diagnostics of neutrinophilic dark matter
}

\author{
Ottavia Balducci, ${ }^{*}$ Stefan Hofmann, ${ }^{\dagger}$ and Alexis Kassiteridis ${ }^{*}$ \\ Arnold Sommerfeld Center for Theoretical Physics, Theresienstraße 37, 80333 München, Germany
}

(Received 8 November 2017; published 2 July 2018)

\begin{abstract}
The standard model of particle physics is extended by adding a purely neutrinophilic dark sector. It is shown that theories which accommodate standard model neutrinos as dark radiation are resurrected. Sterile neutrinos bridge the visible and the dark sector and can keep their mutual interactions effective even after the epoch of big bang nucleosynthesis. This prolonged contact between dark matter and standard model neutrinos solves the well-known small-scale structure problems of the cosmological standard model. The dark sector presented in this work satisfies all experimental and observational constraints from particle physics and cosmology.
\end{abstract}

DOI: 10.1103/PhysRevD.98.023003

\section{INTRODUCTION}

Although much is known about the properties dark matter is required to possess, its precise nature still eludes us. Standard model extensions include particle candidates that are classified as cold and warm dark matter, e.g., sterile neutrinos [1], both of which allow the formation of cosmic large-scale structures in accordance with observations, but at the same time give rise to challenges on small scales known as the "missing satellite," "cusp vs core" and "too big to fail" problems; for an overview of these issues see Ref. [2].

Hierarchical structure formation in cold dark matter $(\mathrm{CDM})$ requires, among other prerequisites, a kinetic decoupling from some radiation component after big bang nucleosynthesis (BBN) [2]; for a general analysis of CDM models with late decoupling see Ref. [3]. Almost ten years ago an extremely interesting idea appeared, questioning whether such a radiation component is required to belong to the dark sector extending the standard model (SM), or whether it could belong to the particle spectrum of the standard model. As a response to this question so-called leptophilic models arose [4] that could address the smallscale crisis. It soon became clear that neither charged leptons [5] nor photons [6] can play such a role due to the strong constraints from observations of the cosmic microwave background (CMB) radiation. The focus turned to

\footnotetext{
*ottavia.balducci@physik.uni-muenchen.de 'stefan.hofmann@physik.uni-muenchen.de

a.kassiteridis@physik.uni-muenchen.de
}

Published by the American Physical Society under the terms of the Creative Commons Attribution 4.0 International license. Further distribution of this work must maintain attribution to the author(s) and the published article's title, journal citation, and DOI. Funded by SCOAP ${ }^{3}$. neutrinophilic theories, where the communication channel with the dark sector is kept effective for a sufficient time duration by SM neutrinos [5]. It was already known that some neutrinophilic theories involving vector mediators were able to solve the small-scale problems [7]. However, the parameter space of these attempts is severely constrained by weak decays of SM bosons, and, as a matter of fact, enjoys no intersection [8] with the parameter space required to address the small-scale crisis. Parallel to this development, neutrinophilic theories with a scalar mediator were also ruled out; see Refs. [9,10]. As stated in Ref. [6], the radiation component that is responsible for keeping cold dark matter in kinetic equilibrium for a sufficiently long period, if existent, should belong to the dark sector extending the standard model.

The scientific objective of this article is a proof of concept for a purely phenomenological model, which resurrects the neutrinophilic theories and provides clear solutions to the large- and small-scale structure formation problems, while respecting all constraints from experiments and consistency considerations, as well as the SM symmetries. To the best of our knowledge, there is no model in the literature that successfully addresses cosmic structure formation, satisfies all these constraints and employs SM neutrinos as the last scattering partners for CDM, while still providing ultraviolet completeness, zero dark photon/photon mixing at one loop [11], which is strongly constrained [6], and compatibility with big bang nucleosynthesis [12].

This article is organized as follows. In Sec. II the standard model is extended by a phenomenological dark sector. Its particle spectrum is justified and the relevant interactions are introduced. The following section is devoted to calculating cross sections and decay rates that are pertinent for the cosmological implications of this model. In Sec. IV we consider the constraints from particle 
physics and cosmology and consider how they restrict the parameter space. This allows us to compute the most important cosmological observables, like relic abundance, decoupling temperature and damping scales, in Sec. V. By comparing these quantities with recent experimental results we give the mass spectrum of the theory in Sec. VI. We summarize the results and conclude in Sec. VII.

\section{SINGLETS IN THE DARK SECTOR}

In this section, the dark sector and its coupling to the visible sector are described in detail.

The proposed extension of the standard model $\mathcal{L}_{\text {ext }}$ consists of a dark sector $\mathcal{L}_{\mathrm{ds}}$ and a neutrino bridge $\mathcal{L}_{\mathrm{nb}}$

$$
\mathcal{L}_{\text {ext }}=\mathcal{L}_{\mathrm{ds}}+\mathcal{L}_{\mathrm{nb}}
$$

The dark sector contains a fermion $F$, a sterile neutrino $n$ and three real scalars $S, \Phi, X$. One notices that this field content admits the same number of degrees of freedom (d.o.f.) as in the original vector neutrinophilic theory [7], which was ruled out. All of these new fields are SM singlets. A summary of the new particles and their charges is presented in Table I.

We postulate that these Lagrangians accommodate the usual canonical kinetic terms

$$
\begin{aligned}
\mathcal{L}_{\mathrm{ds}} \supset & \frac{1}{2} \bar{F}(\mathrm{i} \not \partial-m) F+\frac{1}{2} \bar{n}\left(\mathrm{i} \not \partial-M_{R}\right) n \\
& -\frac{1}{2} S\left(\square+m_{S}^{2}\right) S-\frac{1}{2} \Phi \square \Phi-\frac{1}{2} X\left(\square+m_{X}^{2}\right) X .
\end{aligned}
$$

The cosmologically relevant dimension-four interactions are given by

$$
\mathcal{L}_{\mathrm{ds}} \supset \frac{1}{2} g_{S} \bar{F} S F+\frac{1}{2} \bar{g}_{S} \bar{n} S n+\frac{1}{2} g_{X} \bar{F} X F-\mathcal{V}[S, \Phi] .
$$

Here we note that since the model is based on purely phenomenological reasoning, couplings that do not appear are assumed to be weaker than feeble ones. The last part of the dark sector is a scalar potential $\mathcal{V}[S, \Phi]$ that enables a phase transition in the proposed sector; hence

$$
\mathcal{V}[S, \Phi]=\frac{\ell}{4}\left(\Phi^{2}-\bar{v}^{2}\right)^{2}+\frac{x}{4} \Phi^{2} S^{2}
$$

where $\bar{v}$ is a real parameter.

The communication between the dark sector and the SM is achieved via the sterile neutrino bridge

$$
\mathcal{L}_{\mathrm{nb}}=\mathrm{i} y \bar{L} \sigma_{2} H^{*} n+\text { H.c. }
$$

All mass parameters are positive. Finally, the theory should contain a Majorana mass $m_{L}$ for the SM neutrino to accommodate the final observed neutrino mass, $m_{\nu}$, after a hybrid-type seesaw (type I + II); for a gauge-invariant implementation see Ref. [13].

We now justify the new particles and interactions appearing in $\mathcal{L}_{\mathrm{ds}}$ and $\mathcal{L}_{\text {nb }}$.

The dark matter is assumed to be a SM singlet represented by a Majorana fermion $F$. We choose $F$ to be a Majorana fermion, instead of a Dirac one, just to have a more minimal model; but, in principle, both Majorana and Dirac fermions admit similar properties during the thermal evolution of the theory and thus would be equally good candidates. The construction of the dark sector ensures stability of the dark matter particles, since an accidental $\mathbb{Z}_{2}$ symmetry on the $F$ field arises.

In order to solve the large- and small-scale problems of the $\Lambda \mathrm{CDM}$ cosmology, we introduce Yukawa interactions between the dark matter field and the SM-singlet real scalars $S$ and $X$. The interaction mediated through $S$ provides the self-interacting nature of this theory that solves the small-scale problems, while the late decays of the $X$ particles lead to the desired dark matter relic density.

As already mentioned, a further SM-singlet sterile neutrino $n$ is postulated in Eqs. (2), (3), and (5). The motivation behind it is the following: we want the dark sector to couple solely to neutrinos at tree level, and not to charged leptons. This poses a potential problem, because it violates the gauge group of the left-handed fermions. The sterile neutrino mass could be generated through some dark Higgs model with vacuum expectation value $v_{\mathrm{d}}$, so that $M_{R} \sim v_{\mathrm{d}}^{2} / M_{\tilde{F}}$, where $\tilde{F}$ is some heavier fermion field, such that before the electroweak phase transition the fermionic current is conserved. However, a precise derivation of the origin of this mass is beyond the scope of this paper. The sterile neutrino $n$ is represented by a Majorana fermion and is coupled to the SM neutrinos in the usual manifestly

TABLE I. The particles in the dark sector and their charges: they are total singlets regarding the SM gauge group and carry zero charges. A "+" in the third row, indicates a $\mathbb{Z}_{2}$-even symmetry, while a "-" indicates a $\mathbb{Z}_{2}$-odd one.

\begin{tabular}{lccccc}
\hline \hline & \multicolumn{5}{c}{ Fields } \\
\cline { 2 - 6 } Symmetry & $F$ & $n$ & $S$ & $\Phi$ & $X$ \\
\hline $\mathrm{SU}(3) \times \mathrm{SU}(2) \times \mathrm{U}(1)$ & $(\mathbf{1}, \mathbf{1}, 0)$ & $(\mathbf{1}, \mathbf{1}, 0)$ & $(\mathbf{1}, \mathbf{1}, 0)$ & $(\mathbf{1}, \mathbf{1}, 0)$ & $(\mathbf{1}, \mathbf{1}, 0)$ \\
$\mathbb{Z}_{2}$ & - & + & + & - & + \\
\hline \hline
\end{tabular}




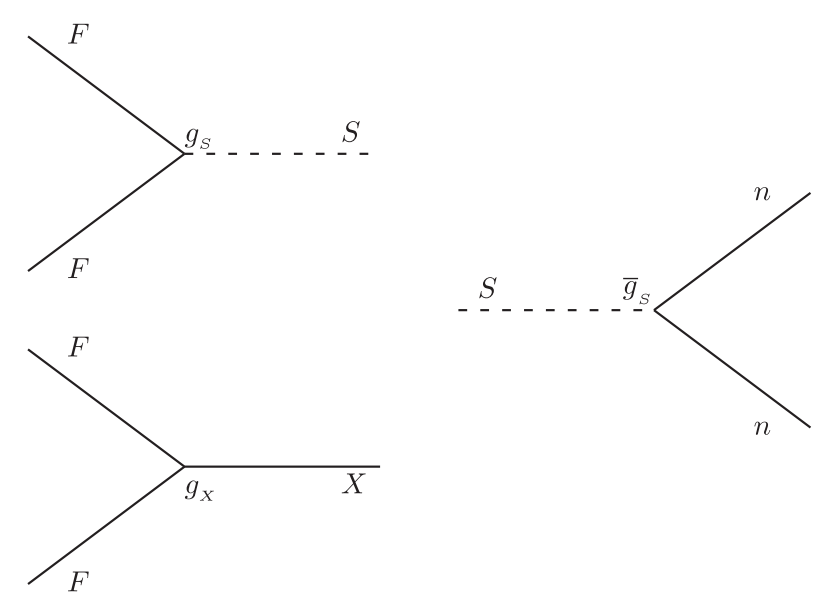

FIG. 1. The Yukawa couplings introduced in the extension to the standard model.

gauge-invariant way introduced in Ref. [14] with coupling $y$ as shown in Eq. (5).

Figure 1 shows the Yukawa interactions between the most important new particles in the dark sector.

The communication between the dark sector and the standard model is then achieved through the gaugeinvariant Yukawa portal given in $\mathcal{L}_{\text {nb }}$. For simplicity, we include in this work only one lepton generation, as the generalization to all generations is straightforward; moreover, we ignore the presence of the Pontecorvo-MakiNakagawa-Sakata matrix. Let us stress already that the sterile neutrino bridge constitutes the longest-lived communication channel between the dark matter and the SM neutrinos. After diagonalizing the neutrino mass matrix, we obtain the Majorana mass eigenstates defined by the corresponding eigenstates of the $p^{2}$ Casimir. With some abuse of notation we call them $n$ and $\nu$ with masses $M_{R}$ and $m_{L}-Y^{2} / 2 M_{R}$ respectively, assuming that $m_{L}, Y \ll M_{R}$, $Y \equiv y v$ and $v \equiv \sqrt{2}\langle H\rangle$.

In other words, the dark sector induces an effective coupling $g_{\nu}$ between the $S$ field and the SM neutrinos after the electroweak phase transition

$$
\frac{g_{\nu}}{2} S \bar{\nu} \nu
$$

with $\left(g_{\nu}\right)^{1 / 2}=\left(\bar{g}_{S}\right)^{1 / 2}\left(\delta r+\mathcal{O}\left(\delta r^{2}\right)\right)$, using the abbreviation $\delta r \equiv Y / \sqrt{2} M_{R}$. This mechanism accommodates naturally effective microcharges between the SM neutrinos and the $S$ bosons. Figure 2 shows the step from the coupling $\bar{g}_{S}$ to the effective coupling $g_{\nu}$. The above effective description is valid at energies below the rest mass of the sterile neutrino $n$, where this field can be integrated out. It is this effective coupling $g_{\nu}$ that is responsible for the late decoupling regime between the dark matter particles and the SM neutrinos and for the correct cutoff masses of the protohalos alleviating the "missing satellite problem" and

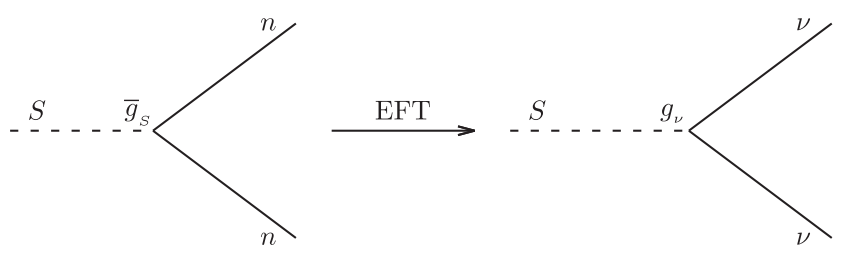

FIG. 2. After the electroweak phase transition the coupling $g_{\nu}$ in the effective theory description (EFT) is obtained.

addressing positively, together with the dark matter selfinteractions, the "cusp vs core" and the "too big to fail" issues.

More precisely, in order to solve the small-scale structure problems efficiently and simultaneously bypass all strong neutrinophilic constraints [8-10], a phase transition in the dark sector should take place, after the protohalo formation. The most minimal realization is achieved after adding an additional d.o.f.; nevertheless, more sophisticated solutions (for example hidden global or local symmetries) could also lead to the desired results, but since $\mathcal{V}[S, \Phi]$ works well, we can keep it as it is. Therefore, we include in the spectrum the real scalar field $\Phi$, which is solely coupled to the singlet $S$ quadratically. Such a scalar potential enables a first-order phase transition at times after big bang nucleosynthesis and could potentially change the mass of the $S$ boson significantly. Furthermore, we denote the temperature of this phase transition by $T_{c}$ and assume $T_{c} \ll T_{\mathrm{EW}}$, where $T_{\mathrm{EW}}$ is the temperature of the electroweak phase transition. This is encapsulated in Fig. 3. For clarity, we denote with $a$ the scalar field after the phase transition has taken place.

The terms of the effective Lagrangian after the symmetry breaking, modulo self-interactions of $a$, that differ from the theory before the breaking are

$$
\begin{aligned}
\left.\mathcal{L}_{\mathrm{ds}}\right|_{\mathrm{SB}} \supset & -\frac{1}{2} S\left(\square+M_{\mathrm{obs}}^{2}\right) S-\frac{1}{2} a\left(\square+m_{a}^{2}\right) a \\
& -\frac{x}{4} a^{2} S^{2}-\frac{x}{2} \bar{v} a S^{2} .
\end{aligned}
$$

After the symmetry breaking, $a$ acquires a mass $m_{a}=\sqrt{2 \ell} \bar{v}$. The singlet boson $S$ admits an observed mass of $M_{\mathrm{obs}}=\sqrt{m_{S}^{2}+x \bar{v}^{2} / 2}$. This allows us to write the critical temperature as $T_{\mathrm{c}} \approx m_{a}^{2} / \sqrt{\ell} M_{\mathrm{obs}}$ [15], assuming
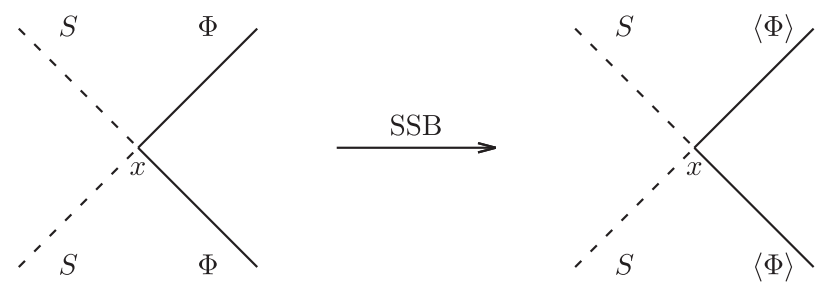

FIG. 3. The scalar field $\Phi$ is introduced with a quartic coupling to $S$ in order to change the mass of $S$ dynamically by a spontaneous symmetry breaking with $\langle\Phi\rangle=\bar{v}$. 


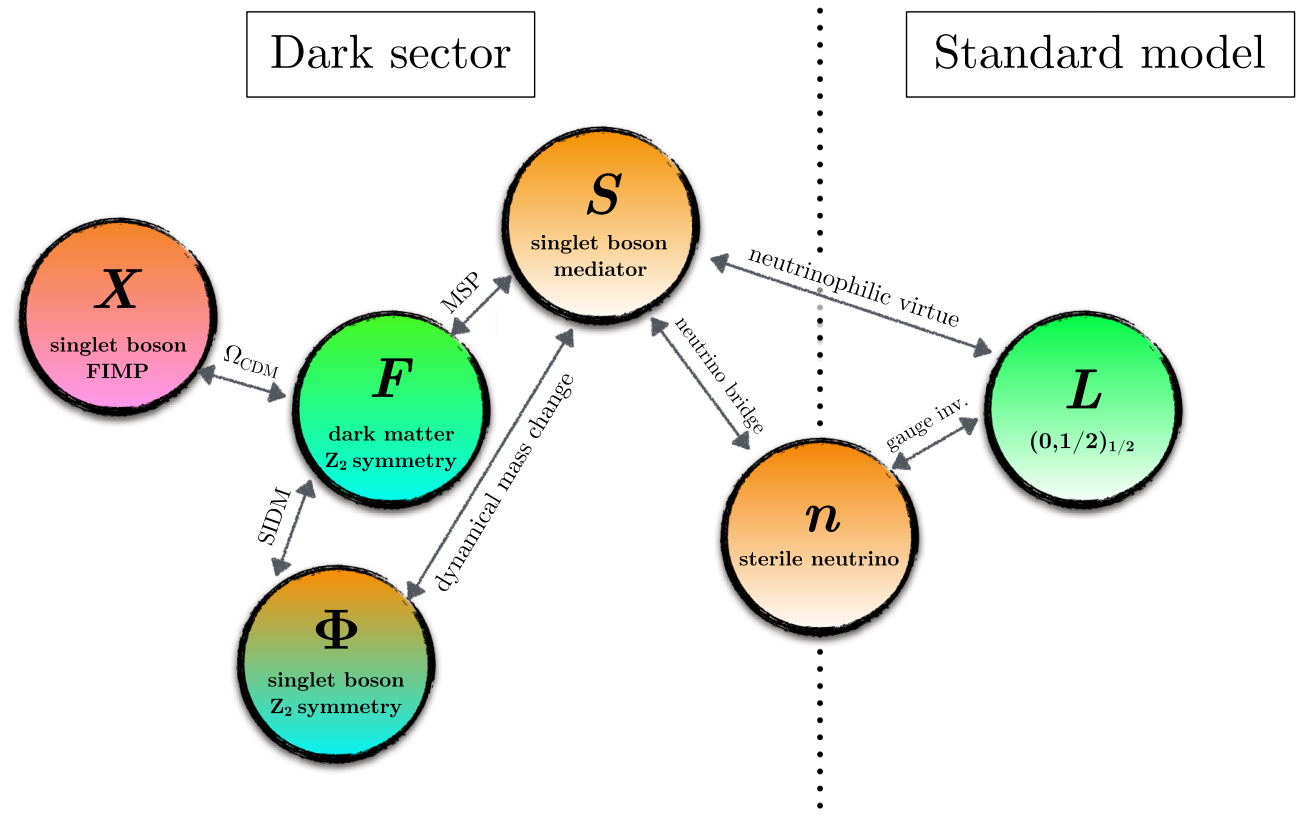

FIG. 4. A representation of the proposed extension to the standard model. The labels on the arrows explain the purpose of each (effective) interaction. Orange colored bubbles indicate that the particle decays, while green colored ones are stable. Particles with an accidental $\mathbb{Z}_{2}$ symmetry are colored in light blue, while purple means that the particle was never in thermal equilibrium with the primordial plasma.

that $2 m_{S}^{2} / x \bar{v}^{2} \ll 1$. Note that both contributions to $M_{\mathrm{obs}}$, $m_{S}^{2}$ and $x \bar{v}^{2}$, are always positive. Figure 4 gives a summary of the newly introduced particles and the relations between them.

Before concluding this section, we present the proposed mass spectrum. We assume the following mass hierarchy in the dark sector:

$$
m_{X} \gg M_{R}>m, m_{h} \gg M_{\mathrm{obs}} \gg m_{S}, m_{a}, m_{\nu} .
$$

For the purpose of this work, we decided to remain agnostic about the origin of the initial boson and dark matter masses and, although achievable, a self-consistent completion of the dark sector is certainly beyond the scope of this paper. Such an arrangement allows various decays, like for example $n \rightarrow h \nu, S \rightarrow \nu \nu, X \rightarrow F F$. Note that the relation of $m_{a}$ with the other masses appears as a natural consequence of requiring a consistent thermal evolution of the theory. Furthermore, the mass of $S$ before the symmetry breaks, $m_{S}$, can be thought of as a $t$-channel regulator in order to simplify the calculations.

\section{CROSS SECTIONS AND DECAY RATES}

This section is devoted to the presentation of cross sections and decay rates relevant for particle physics and structure formation processes in the primordial Universe, in which the dark sector participates.

It is useful to introduce the dark sector Fermi constant of the theory of neutrino scattering $G_{D} / \sqrt{2} \equiv g_{S} g_{\nu} / 4 m_{S}^{2}$.
Annihilation into singlets $S$ implies a sink term in the dark matter abundance that is characterized by the $p$-wave cross section

$$
\left\langle v_{\mathrm{rel}} \sigma_{\mathrm{ann}}\right\rangle=\frac{3}{8} \frac{\pi \alpha^{2}}{m^{2}}\left\langle v_{\mathrm{rel}}^{2}\right\rangle,
$$

where $\langle\ldots\rangle$ denotes the thermal average using relative velocities, $\alpha=g_{S}^{2} / 4 \pi$. In principle, if $m>M_{R}$ the $F$ 's annihilate to $n$ 's; however, this possibility is excluded by the assumed mass hierarchy (8).

Finally, the dark matter abundance is created by $X$ decays with a rate

$$
\Gamma_{X \rightarrow F F}=\frac{g_{X}^{2}}{16 \pi} m_{X}
$$

This concludes the brief presentation of the dominant sinks and sources for the dark matter abundance in the temperature regime $m \gg T \gg m_{S}$, where $T$ is the average photon temperature.

Elastic scatterings involving dark matter particles determine their kinetic properties and allow to quantify their deviation from local thermal equilibrium in terms of viscous processes sourcing entropy production. As mentioned above, the longest running channel in this respect is the one with SM neutrinos as scattering partners. At temperatures $m_{S} \gg T \gg m_{\nu}$, and at lowest order in the Fermi constant, the averaged momentum transfer elastic cross section, defined by $\sigma_{T} \equiv \int \mathrm{d} \Omega(1-\cos \theta) \mathrm{d} \sigma_{\text {el }} / \mathrm{d} \Omega$, is given by 


$$
\left\langle v_{\mathrm{rel}} \sigma_{T}\right\rangle=\frac{80 \zeta(5)}{\pi \zeta(3)} G_{D}^{2} T^{2}\left(\frac{T_{\nu}}{T}\right)^{2}
$$

after applying the equipartition theorem. Note that in the above expression all neutrino masses have been neglected. $T_{\nu}$ denotes the neutrino temperature. The elastic scattering of $S$ particles to neutrinos is extremely suppressed. Indeed, although one can integrate out the sterile neutrino field to obtain $g_{\nu}$ for the physical neutrinos, the internal two-point functions include sterile neutrino propagators, which are not bare propagators but must be perturbatively corrected with internal neutrinos. These rates are suppressed by a factor of $g_{\nu}^{4}$ for the IR-dominant part.

The numerical solutions in various regimes of the momentum transfer cross section for fermions can be found in Ref. [16] and for the classical limit, $m v / m_{S} \gg 1$, we cite semianalytic formulas for the corresponding total cross sections in different kinematical regimes:

$$
\sigma_{T} \approx \begin{cases}\frac{2 \pi}{m_{S}^{2}} \beta^{2} \ln \left(1+\beta^{-2}\right), & \beta \lesssim 1, \\ \frac{\pi}{m_{S}^{2}}(\ln (2 \beta)-\ln (\ln (2 \beta)))^{2}, & \beta \gtrsim 1\end{cases}
$$

where $\beta \equiv 2 \alpha m_{S} /\left(m v_{\text {rel }}^{2}\right)$. This dependence seems to be of vital importance in order to resolve small-scale anomalies that are typically present when structures form in non-selfinteracting dark matter (non-SIDM). Self-interactions among the dark matter particles can be mediated via $X$ as well. In this case, however, there is no significant Sommerfeld enhancement.

\section{PARAMETER CONSTRAINTS}

We proceed to the discussion of the constraints on the dark sector arising from particle physics and cosmology.

\section{A. Constraints from particle physics}

The dark matter particles $F$ are assumed to be stable and produced in local thermal equilibrium. Then partial-wave unitarity of the scattering matrix bounds the annihilation cross section in the primordial Universe, which in turn bounds the relic abundance and the universal mass of the dark matter [17], $m<\mathcal{O}(300) \mathrm{TeV}$. The effective number of neutrino generations as measured by the Planck satellite implies a lower mass bound $m>\mathcal{O}(1) \mathrm{MeV}$. Note that this bound holds precisely for the dark matter introduced in the dark sector, since it is kept in local thermal equilibrium for an extended period via elastic scatterings with SM neutrinos. A priori there are no such bounds on the sterile neutrino mass $M_{R}$ or on the masses $m_{S}, m_{X}$, other than the imposed mass hierarchy (8). Lower bounds on these mass parameters do, however, arise in scenarios where the sterile neutrinos and the dark bosons are kept in local thermal equilibrium with the SM neutrinos after their respective kinetic decoupling from other SM species. An extensive list of a variety of constraints on lepton boson interactions can be found in Ref. [5].

Let us turn to the particle physics constraints on the dark sector couplings. The couplings $g_{S}, \bar{g}_{S}$ and $g_{X}$ are assumed to respect the perturbative domain. In addition the coupling in the Yukawa bridge is bounded by $Y^{2} / 2 M_{R} \sim m_{L} \lesssim$ $5 \mathrm{GeV}$ [13]. Accordingly, in this work we set the upper bound $\alpha \leq 1 / 2$, which in turn implies an upper bound on $m \approx 1.5 \mathrm{TeV}$, as will be shown below. Note that the dark sector does not facilitate a coupling between the dark bosons and charged leptons. Therefore, $g_{\nu}$ is only subject to restrictions arising from the three-body decays of $Z^{0}, W^{ \pm}$ and from the $K^{ \pm}$decays, although such constraints are less tight than those coming from cosmology. Furthermore, we anticipate that such constraints should not be as strong as the light vector-neutrino interaction bounds [8], due to the absence of a longitudinal contact term.

Consider the invisible three-body decay $Z^{0} \rightarrow \nu \nu S$ with rate $\Gamma_{Z^{0} \rightarrow \nu \nu S} \approx 0.18 \mathrm{GeV} g_{\nu}^{2}\left(N_{\nu} / 3\right)$ for an observed $S$ boson mass parameter of $\mathcal{O}(10) \mathrm{MeV}$. The experimental error on $Z^{0}$ decays is $0.0023 \mathrm{GeV}$ [18]. This allows to constrain the effective neutrino coupling by $g_{\nu} \leq 0.12 / \sqrt{N_{\nu}}$. The result does not depend strongly on $M_{\mathrm{obs}}$. Note that if $S$ decays sufficiently fast, then the four-body decay $Z^{0} \rightarrow 4 \nu$ is dominant, which lightens the previous constraint. The dark sector allows the decay $W \rightarrow \nu e S$ as well. The experimental error on the decay of charged electroweak gauge bosons is $0.042 \mathrm{GeV}$ [18]. For $M_{\text {obs }} \sim \mathcal{O}(10) \mathrm{MeV}$ this implies $g_{\nu} \lesssim \mathcal{O}(1)$, which is not a significant constraint in our context. Finally, the constraint on the effective neutrino coupling arising from $K^{ \pm}$decays is more restrictive. In addition to the standard decay $K \rightarrow \nu \mu$, the channel $K \rightarrow \nu \mu S$ is available as well. For masses $m_{S}$ of order $\mathcal{O}(10) \mathrm{MeV}$ and energies between 165.5 and $205.5 \mathrm{MeV}$ for the outgoing muon, the relative rate is almost constant and can be explicitly written [19] as

$$
\frac{\Gamma_{K \rightarrow \nu \mu S}}{\Gamma_{K \rightarrow \nu \mu}}=7.4 \times 10^{-4} g_{\nu}^{2}
$$

The experimental bound on this ratio is $3.6 \times 10^{-6}$, implying a bound on the effective neutrino coupling $g_{\nu} \leq 7 \times 10^{-2}$.

Note that, contrary to the situation analyzed in Ref. [8], there is no constraint from the elastic $\nu e \rightarrow \nu e$ scattering, since the dark singlets in the dark sector are decoupled from charged leptons at tree level. Additional bounds on $g_{\nu}$ due to lepton number violation and mesonic decays were computed in Ref. [9]. However, they are less tight than the cosmological constraints, which will be discussed in the next section. Since $g_{\nu} \lesssim 0.12 / \sqrt{N_{L}}$ and $\Gamma_{\text {el }} \sim N_{L} g_{\nu}^{2}$, the extremal result for $\Gamma_{\mathrm{el}}$ does not depend on the actual number of neutrinos coupled effectively to the singlet boson; however, if $N_{S}$ denotes the number of identical $S$ bosons which are present, then $g_{\nu}$ scales with $1 / \sqrt{N_{S}}$. 
Further bounds on self-annihilation thermal averaged cross sections of $F$ 's to neutrinos can be found in Ref. [20].

\section{B. Constraints from cosmology}

In this part, we study the constraints on the couplings $x, g_{\nu}$ and $g_{X}$, which arise from astrophysical observables.

The desired value of $M_{\mathrm{obs}}$ lies in the sub-GeV/multiple$\mathrm{MeV}$ interval in order to solve the small-scale issues. Furthermore, $m_{S}$ should lie in the (sub-)keV range to enable a late kinetic decoupling. Therefore, we discuss only the case, where the phase transition in the dark sector takes place after the kinetic decoupling of the $F$ particles from the neutrinos; otherwise, the relevant interactions are highly suppressed. When the condensation of $\Phi$ happens after $T_{\mathrm{kd}} \sim \mathcal{O}\left(10^{2}\right) \mathrm{eV}$, the $S$ particles annihilate rapidly to $a$ particles, as long as $m_{a} \ll M_{\text {obs }}$. We demand that at the phase transition $S$ and $\Phi$ are in local thermal equilibrium. This condition implies the following constraint for the coupling strength between $\Phi$ and $S$ :

$$
x \gtrsim 10^{-13}\left(\frac{T_{\mathrm{c}}}{100 \mathrm{eV}}\right) .
$$

If the mass parameters $m_{a}$ and $m_{S}$ of $a$ and the $S$ bosons respectively were below $\mathcal{O}(1) \mathrm{MeV}$, the presence of these particles in the primordial plasma would modify big bang nucleosynthesis. Furthermore, if these mass parameters were close to the temperature of the primordial plasma at recombination, the cosmic microwave background radiation would carry a fingerprint of the dark sector. However, if the light particles of the dark sector and the neutrinos freeze in after the end of big bang nucleosynthesis, at around $30 \mathrm{keV}$, the impact is minimized. This leads to a conservative upper bound for the effective neutrino coupling $g_{\nu}^{2} \lesssim 10^{-12}$ using the IR-dominant part of the elastic cross sections in the absence of a fundamental cubic scalar interaction in the dark sector. Furthermore, an important bound of $g_{\nu} \gtrsim 1.6 \times 10^{-6} \mathrm{MeV} / M_{\text {obs }}$ arises for $M_{\text {obs }} \sim \mathcal{O}(1) \mathrm{MeV}$, as computed from the luminosity and deleptonization arguments regarding the observation of SN1987A in Ref. [10]. In this model values of $M_{\text {obs }} \approx$ $50 \mathrm{MeV}$ alleviate the cusp vs core and the too-big-to-fail problems together with a suitable set of $\left\{m, g_{X}\right\}$. Therefore, the final constraint on $g_{\nu}$ reads $10^{-15} \lesssim g_{\nu}^{2} \lesssim 10^{-12}$. Taking the saturation limit of this relation, the effective neutrino coupling can be eliminated as a free parameter; hence, we consider a typical value of $g_{\nu}^{2}=10^{-13}$ in this work.

For simplicity we assume that the light dark sector particles are in local thermal equilibrium after big bang nucleosynthesis and before the phase transition takes place. After the condensation of $\Phi$, the dark $a$ emerges as a massive propagating d.o.f. The metastable massive $a$ 's contribute to the energy density of the Universe by a factor of $\Omega_{a} h^{2} \approx m_{a} / 300 \mathrm{eV}$. Nevertheless, the condition that
$\Omega_{a} h^{2}$ be small enough is easily fulfilled, since $m_{a}$ lies well below the eV scale.

Since the scalar field $\Phi$ couples directly only to the $S$ boson, we turn our attention to the properties of the interactions between $S, \Phi$ and the standard model. To test the above construction, we calculate the deviation of the effective neutrino d.o.f., which parametrizes the energy density of the Universe, in different periods of its evolution. This is encapsulated in the following definition, assuming that the neutrinos already decoupled at $T_{\nu D}=2.3 \mathrm{MeV}$ [21]:

$$
\left.\Delta N_{\mathrm{eff}}\right|_{\mathrm{BBN}}=N_{\nu} \frac{\rho_{S+\Phi}}{\rho_{\nu}} .
$$

$\rho_{i}$ is the equilibrium energy density of the $i$ th particle species with initial conditions defined at $T_{\nu D}$ and including all available d.o.f. This number is important because it parametrizes the cosmic energy budget and can therefore be probed with high precision. Let us introduce the abbreviation $\left.\varepsilon\right|_{T_{\nu} D} \equiv\left(T_{S} / T_{\nu}\right)^{3}$.

Examining the parameter space we find that a common value of $\varepsilon \sim 0.1$ is compatible with big bang nucleosynthesis [22] and cosmic microwave background [23] $1 \sigma$ measurements. Indeed one obtains $\left.\Delta N_{\text {eff }}\right|_{\text {BBN }} \approx 0.05$ and $\left.\Delta N_{\mathrm{eff}}\right|_{\mathrm{CMB}} \approx-0.02$ assuming that $T_{\mathrm{c}}>T_{\text {rec }}$. This may also explain the recent tension about the decrease of the deviation of the effective neutrino number from BBN to CMB-based measurements. A difference $\left.\Delta N_{\text {eff }}\right|_{\text {CMB }}-$ $\left.\Delta N_{\text {eff }}\right|_{\text {BBN }}<0$ is possible in the underlying theory. However, if $T_{\mathrm{c}}<T_{\text {rec }}$ and at the same time all light d.o.f. are not in local thermal equilibrium with neutrinos at the recombination period, then $\left.\Delta N_{\text {eff }}\right|_{\mathrm{CMB}} \approx 0.05$; therefore, for optimal big bang nucleosynthesis/cosmic microwave background compatibility the phase transition should happen before the recombination period with all light particles in local thermal equilibrium. Moreover, we make the bound less tight by demanding that $N_{\text {eff }}+\Delta N_{\text {eff }} \lesssim 3.5$ $[4,23]$. This sets an upper bound to the number of singlet bosons, $N_{S} \leq 8$.

It is crucial that the $S$ boson should decouple from the primordial plasma early enough, when all or almost all the d.o.f. of the standard model are accessible. Quantitatively this means that the $S$ boson decoupling from the SM plasma should take place before the QCD phase transition, $T_{\text {freeze-out }}>T_{\mathrm{QCD}}$, to obtain an allowed value of $\varepsilon \lesssim 0.2$. This corresponds to a lower bound for $M_{R}$ with respect to the couplings of the theory. We obtain up to $\mathcal{O}(1)$ factors,

$$
\left(\frac{g_{\nu}}{10^{-6}}\right)\left(\frac{\bar{g}_{S}}{1}\right)\left(\frac{\mathrm{TeV}}{M_{R}}\right) \lesssim \mathcal{O}(1)
$$

In other words, light dark scalar bosons went through kinetic decoupling from the SM species at temperatures $T_{S D} \gg T_{\nu D}$ due to their IR-suppressed part of the cross section for elastic scatterings with SM neutrinos. 
For instance, $T_{S D}=\mathcal{O}\left(10^{6}\right) T_{\nu D}$ for $M_{R}=\mathcal{O}(10) \mathrm{TeV}$, $\bar{g}_{S}=0.05$ and $g_{\nu}^{2} \approx 10^{-13}$. In this example kinetic decoupling from the SM species happens at $T_{S D} \approx 0.5 \mathrm{TeV}$.

The above discussion does not involve masses larger than $T_{\nu D}$ and as long as $\tau_{S} \ll \tau_{\mathrm{BBN}}$ no particular constraints arise. It turns out that the kinetic properties of the dark sector depend on the mass of the light dark bosons relative to the neutrino decoupling temperature, as will be worked out in great detail in the next section. Anticipating distinct kinetic regimes, the dark sector will be referred to as model $\mathcal{D S} \mathcal{S}_{\mathrm{M} 1}$ if $m_{S} \leq \mathcal{O}(1) \mathrm{keV} \ll T_{\nu D}$, and as model $\mathcal{D} \mathcal{S}_{\mathrm{M} 2}$ if $m_{S}>T_{\nu \mathrm{D}}$. Let us briefly turn to $\mathcal{D} \mathcal{S}_{\mathrm{M} 2}$ and consider the modification of the effective neutrino d.o.f. in this case,

$$
\Delta N_{\mathrm{eff}}=\frac{60}{7 \pi^{4}} \int_{x_{\mathrm{SBBN}}}^{\infty} \mathrm{d} z z^{2} \frac{\sqrt{z^{2}-x_{S \mathrm{BBN}}^{2}}}{\exp (z)-1},
$$

where $x_{\text {SBBN }}=m_{S} / T_{\mathrm{BBN}}$. As an example, for $m_{S}=$ $10.5 \mathrm{MeV}$ we obtain $N_{\text {eff }}=3.15$, which matches perfectly with the value inferred from the Planck measurements [23]. At the $2 \sigma$ level, these also impose a lower bound for the mass of the light dark bosons of $m_{S}>3.5 \mathrm{MeV}$. In other words, the light dark scalar bosons in $\mathcal{D} \mathcal{S}_{\mathrm{M} 2}$ are roughly 6 orders of magnitude heavier compared to those in the $\mathcal{D} \mathcal{S}_{\mathrm{M} 1}$ model. In the next section it will be shown how this renders $\mathcal{D} \mathcal{S}_{\mathrm{M} 2}$ less attractive from a phenomenological point of view.

Finally, the feebly interacting massive boson $X$ should have a decay channel into dark matter flavors after chemical decoupling at $x_{\mathrm{f}}$, and this channel should close before the epoch of big bang nucleosynthesis. Using the corresponding decay rate (10), the following interval for the coupling of the massive scalar $X$ to the dark matter can be inferred:

$$
\begin{aligned}
4 & \times 10^{-5}\left(\frac{T_{\nu D}}{2.3 \mathrm{MeV}}\right)\left(\frac{g_{*}}{10.75}\right)^{1 / 4} \\
& \lesssim\left(\frac{g_{X}}{3 \times 10^{-10}}\right)\left(\frac{m_{X}}{\mathrm{PeV}}\right)^{1 / 2} \lesssim\left(\frac{30}{x_{\mathrm{f}}}\right)\left(\frac{g_{*}}{108.75}\right)^{1 / 4}\left(\frac{m}{\mathrm{TeV}}\right),
\end{aligned}
$$

where $g_{*}$ counts the effective d.o.f. in the primordial plasma. This concludes the discussion of the coupling constraints.

\section{COSMOLOGICAL OBSERVABLES}

In this section we calculate the relic abundance of the dark matter candidates in the spectrum of the dark sector, their kinetic decoupling temperature, both for $\mathcal{D S} \mathcal{S}_{\mathrm{M} 1}$ and $\mathcal{D} \mathcal{S}_{\mathrm{M} 2}$, and evaluate the characteristic damping scales implied by kinetic decoupling and free streaming of the dark matter candidates together with their cross sections for self-interaction. Collisional and collision-free damping impact structure formation and determine the properties of the typical first protohalos. Finally, we investigate whether the dark sector allows to resolve challenges for $\Lambda \mathrm{CDM}$ posed by the formation of cosmic structure as it is observed. In this paper, we neglect a possible second period of dark matter annihilation and the possible formation of dark matter bound states [6]. Alternatively, one can consider out of equilibrium dark matter production from late decays. We are only interested in investigating the possibility of the existence of a simple field-theoretical model, which alleviates the large and small structure problems. We note that all temperatures are given in the photon plasma frame, unless stated differently.

\section{A. Dark matter relic abundance}

Let us first assume that the $F$ fields are the dominant dark matter population, with annihilations into light singlet bosons as the dominant sink for this population towards chemical decoupling. $\alpha$ is fixed by requiring that the observed relic density is correctly retrieved in the unbroken phase. The defining equation of the dark matter distribution function $f(\mathbf{p}(t))$ per d.o.f. of the underlying field in a Friedmann-Robertson-Walker universe yields

$$
(L-C)[f](\mathbf{p})=0
$$

where $L:=p^{0}\left(\partial_{t}-H \mathbf{p} \cdot \nabla_{\mathbf{p}}\right)$ is the Liouville operator, which gives the change of $f$ with respect to an affine parameter along a geodesic, and $C$ is the collision term. We can simplify the Boltzmann equation by taking all the participating particles up to the dark matter ones to admit equilibrium thermal distributions. The Boltzmann equation for nonrelativistic chemical decoupling leads to the change of the number density $n$ of dark matter particles per entropy density $s$, denoted by $Y$, with respect to the temperature $T \equiv m / x$ according to the Riccati-type equation,

$$
\frac{\mathrm{d} Y_{F}}{\mathrm{~d} x}=-\frac{E}{x^{\ell}}\left(Y_{F}^{2}-Y_{F}^{\mathrm{eq} 2}\right)
$$

where the efficiency is given by $E \equiv s(x)\left\langle v_{\text {rel }} \sigma_{\text {ann }}\right\rangle /$ $\left.H(x)\right|_{x=1}$, and $\ell=3$ for $p$-wave annihilations, assuming approximately massless final states [more precisely $\left.m_{S} /(m / x) \ll 1\right]$. We define the chemical freeze-out value $x_{\mathrm{f}}$ by demanding that

$$
\left\langle v_{\text {rel }} \sigma_{\text {ann }}\right\rangle n_{F}^{\mathrm{eq}}\left(x_{\mathrm{f}}\right) \stackrel{!}{=} H\left(x_{\mathrm{f}}\right) .
$$

The exact solution of this equation is given in terms of the Lambert- $W$ function, but a sufficiently good approximation can be found iteratively. Already after one iteration 


$$
\begin{aligned}
& x_{\mathrm{f}}=\ln (C)-\frac{\frac{1}{2} \ln (\ln (C))}{1+\frac{1}{2} \ln ^{-1}(C)}, \\
& C=\sqrt{\frac{45}{32}} \frac{2 M_{\mathrm{Pl}} m}{\left.\sqrt{g_{*}}\left(T_{\mathrm{f}}\right)\left\langle v_{\mathrm{rel}} \sigma_{\mathrm{ann}}\right\rangle^{-1}\right|_{x=1}} .
\end{aligned}
$$

This assumes that the number of relativistic d.o.f. varies slowly with the temperature towards chemical decoupling. Inserting Eq. (22) into Eq. (20), the differential equation for $Y_{F}$ can be solved numerically for the coupling constant $\alpha$. Requiring that $n_{F}\left(x_{\mathrm{f}}\right) \gg n_{F}^{\mathrm{eq}}(\infty)$, and in the absence of feeble couplings, we obtain for the relic abundance of the dark matter population as a function of $m$ and $\alpha$,

$$
\Omega_{F} h^{2} \approx 0.12\left(\frac{\alpha}{0.1}\right)^{-2}\left(\frac{m}{\mathrm{TeV}}\right)^{2}
$$

The self-annihilation cross section $\sigma_{\text {ann }}$ is well below the experimental sensitivity [20]. Note that Eq. (23) has been obtained assuming $g_{S} \gg g_{\nu}$ in order to neglect annihilations into SM neutrinos. We also included the Sommerfeld effect [7], which gives corrections of $\mathcal{O}(1)$ to the above result.

Let us stress that the constraints and the relic abundance (23) fix $g_{S}$ completely. However, in both models in order to resolve the missing satellite problem, typically larger values of $\alpha$ are needed for such masses; as a consequence, a different (primary or subsidiary) mechanism to populate the dark matter is required, which is still compatible with the framework above. The only option left in the dark sector is to depart from the weakly interacting massive particle (WIMP) scenario as the exclusive dark matter production mechanism and consider the out-of-equilibrium decay of the heavy scalar $X$. The number of $X$ particles per entropy varies with the temperature $T=m_{X} / x$ as described by

$$
\frac{\mathrm{d} Y_{X}}{\mathrm{~d} x} \approx \frac{3}{8 \pi^{5}} \sqrt{\frac{5}{\pi}} \frac{M_{\mathrm{P}} \Gamma_{X \rightarrow F F}}{g_{*}^{3 / 2}\left(T_{\mathrm{f}}\right) m_{X}^{2}} x^{3} K_{1}(x),
$$

where $K_{1}$ is the modified Bessel function of the second kind with index one. The $\ell=-3$ behavior is a characteristic property of models with feebly interacting massive particles (FIMPs). From this we find for the $F$ relic abundance,

$$
\begin{aligned}
\Omega_{F} h^{2} \approx & 0.12\left(\frac{g_{X}}{8 \times 10^{-11}}\right)^{2}\left(\frac{114}{g_{*}\left(T_{\mathrm{f}}\right)}\right)^{3 / 2} \\
& \times\left(\frac{m}{\mathrm{TeV}}\right)\left(\frac{\mathrm{PeV}}{m_{X}}\right)
\end{aligned}
$$

It is very promising that this scenario, for values of $g_{X} \gtrsim 10^{-9}$, is perfectly compatible with the constraints on the $g_{X}$ coupling (18), as can be seen in Fig. 5. Concluding, in the presence of multiple heavy bosons, smaller couplings are needed, which allow smaller dark matter masses for higher values of $m_{X}$ due to Eq. (18).

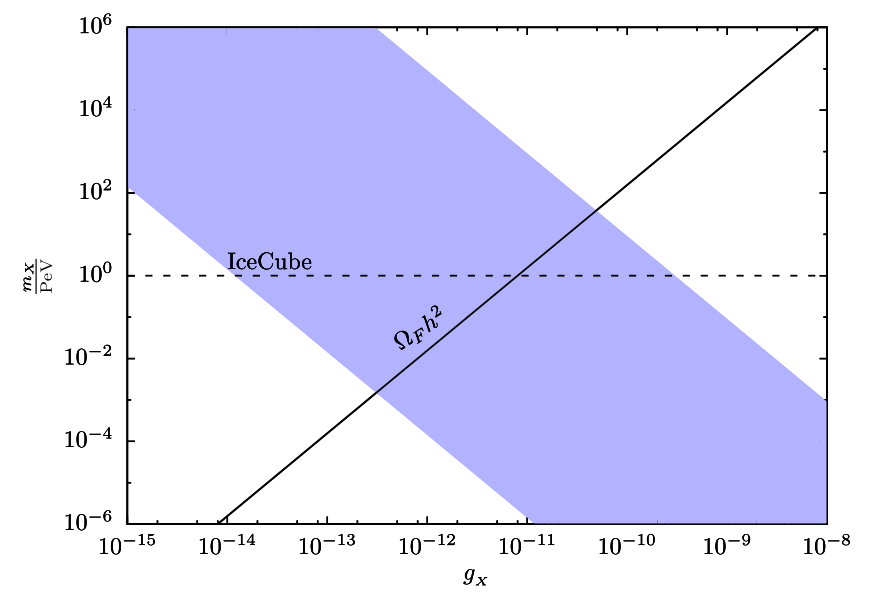

FIG. 5. Constraints on the relation between the coupling constant $g_{X}$ and the mass of the heavy scalar $X$. The shaded region represents the parameter space allowed by Eq. (18), while the solid curve comes from the condition for the correct relic density given in Eq. (25). The dashed line represents the recent IceCube measurements of ultra-energetic neutrinos in the $\mathrm{PeV}$ regime. A dark matter mass of $m=1 \mathrm{TeV}$ is assumed.

The same can be achieved after using Dirac fermions for $F$ and/or upgrading $X$ to a vector boson.

\section{B. Decoupling temperatures}

As calculated above, chemical decoupling of the dark particles happens long before the SM neutrinos decouple. However, this is not the case for the end of efficient momentum exchange: the kinetic coupling of dark matter to neutrinos is active for much longer. In order to have a phenomenologically viable scenario, kinetic decoupling should be scheduled for times long after big bang nucleosynthesis, but still before the $\sim 100 \mathrm{eV}$ epoch, due to the constraints from the Lyman- $\alpha$ measurements [24]. We use simply the constraints from the general overview [3] and not the most recent ones [25], since such constraints are described as overly restrictive; see Ref. [26].

A rough but sufficiently accurate estimate for the temperature $T_{\mathrm{ls}}$ of the typical last elastic contact between the dark matter and the SM neutrinos, which represents the longest active communication channel, is obtained by equating the corresponding elastic scattering rate $\Gamma_{\mathrm{el}} \equiv$ $\left\langle v_{\text {rel }} \sigma_{\mathrm{el}}\right\rangle n_{\nu}^{\mathrm{eq}}$ with the Hubble expansion rate. For example, assuming three generations of SM neutrinos and $m_{S}<\mathcal{O}(1) \mathrm{keV}$, we find $T_{\mathrm{ls}} \approx T_{\text {eq }}$, where $T_{\text {eq }}$ denotes the temperature of matter-radiation equality. This characterizes the typical spatial hypersurface from which dark matter enters the free-streaming regime.

$T_{\mathrm{kd}}$ is the temperature at which the dark sector kinetically decouples from the standard model, when the elastic interactions between the dark matter particles and the neutrino generations cease to sustain local thermal equilibrium [27]. In model $\mathcal{D} \mathcal{S}_{\mathrm{M} 1}$ it depends on the parameters 
$\left\{g_{X}, m_{S}, m, g_{\nu}\right\}$. Hence, the temperature at kinetic decoupling is not completely fixed by the relic dark matter abundance. Moreover, these parameters allow to accommodate other constraints, like the one on $\left\langle\sigma_{T} / m\right\rangle$, to address the cusp vs core problem.

We note that $T_{\mathrm{kd}}$ is not very sensitive to small changes of $m_{S}<\mathcal{O}(1) \mathrm{keV}$, but it changes rapidly with respect to deviations of the neutrino effective coupling. Numerically, using the rate of the averaged momentum transfer elastic cross section as in Ref. [5] and equating it to the Hubble rate, we obtain $T_{\mathrm{kd}} \lesssim 0.6 \mathrm{keV}$ and at the same time the variable set $\left\{g_{S}, m, m_{S}, g_{\nu}\right\}$ fulfills all previous constraints. If a larger value of $\alpha$ is needed, then the FIMP scenario comes into play and cures this issue. An elegant instantaneous kinetic decoupling can be achieved by setting the critical temperature equal to the desired $T_{\mathrm{kd}}$.

\section{Damping scales}

Eventually, around a temperature $T_{\mathrm{kd}}$, elastic scattering processes between dark matter and SM neutrinos happen too infrequently to sustain local thermal equilibrium. Since the neutrino abundance is much larger than the dark matter one, every nonrelativistic dark matter particle will interact a large number of times with the relativistic neutrinos until the time of kinetic decoupling. On the other hand, hardly any neutrinos will ever interact with a dark matter particle. Around $10^{10}$ interactions between dark matter and neutrinos are needed for a $\mathcal{O}(1)$ momentum transfer. This is easily achieved since the abundances of the two types of particles are so different from each other and since there is a long enough period of time until kinetic decoupling [28]. For this reason, any structure smaller than $M_{\mathrm{d}}=$ $(4 \pi / 3) \rho_{\mathrm{m}}\left(T_{\mathrm{kd}}\right) / H^{3}\left(T_{\mathrm{kd}}\right)$ will be destroyed before $T_{\mathrm{kd}}$ [29]. We thus find the following estimate of the characteristic damping mass:

$$
M_{\mathrm{d}} \approx 2.7 \times 10^{8} \frac{g_{* \mathrm{~s}}\left(T_{\mathrm{kd}}\right)}{g_{*}^{3 / 2}\left(T_{\mathrm{kd}}\right)}\left(\frac{\mathrm{keV}}{T_{\mathrm{kd}}}\right)^{3} M_{\odot},
$$

where $g_{* \mathrm{~s}}(T)$ and $g_{*}(T)$ denote the effective number of relativistic d.o.f. contributing to the entropy and energy density at temperature $T$ respectively.

A relevant independent quantity is the free-streaming length of dark matter particles [30]. However, we expect that the acoustic damping is much more efficient than free streaming for very late decoupling temperatures, since the latter has a $1 / T_{\mathrm{kd}}^{3 / 2}$ dependence in contrast to the $1 / T_{\mathrm{kd}}^{3}$ dependence of $M_{\mathrm{d}}$. The mass of the smallest possible protohalo, that could be formed, is found by taking the largest of the above two masses. A possible solution to small-scale abundance problems of $\Lambda$ CDM cosmology, i.e., the missing satellite problem, can be found after suppressing the power spectrum at scales as large as that of dwarf galaxies [31], which is provided by damping masses of order $\log _{10}\left[M_{\mathrm{d}} / M_{\odot}\right] \gtrsim 9$ and not larger than $\sim 10^{10} M_{\odot}$ as stated in Refs. [3,5,24,31,32]. For a thorough discussion see Ref. [33]. It should be clear that if a stronger upper bound is activated, then it is much easier to find a common parameter set to achieve such kinetic decoupling temperatures; nevertheless, we do not examine such a case in this work.

In model $\mathcal{D} \mathcal{S}_{\mathrm{M} 1}$, the mass scale of acoustic damping [34] can be easily fixed at $10^{10} M_{\odot}$. In model $\mathcal{D S} \mathcal{S}_{\mathrm{M} 2}$, the cutoff masses are much smaller, namely we approximately obtain $M_{\mathrm{d} D \mathcal{S}_{\mathrm{M} 2}} \approx 5 \times 10^{3} M_{\odot}$. These values are far from promising. Although they allow protohalo masses that are not excluded by current collider and direct search constraints, they do not provide a solution to the missing satellite problem. We note that for neutralinos in the minimal supersymmetric SM the predicted masses are around the Earth's mass [30]. Such masses are well below the masses that the current numerical simulations studying the largescale structures can resolve [28].

Concluding, we notice that the model $\mathcal{D} \mathcal{S}_{\mathrm{M} 2}$ is ruled out by astrophysics, if one assumes that the astrophysical constraint of order 9 is actually the lower bound. This does not alleviate the enduring missing satellite problem respecting the Planck measurements as stated in Ref. [31]; in other words, at least $m_{S}<\mathcal{O}(1) \mathrm{keV}$ is needed in order to generate masses near $10^{10} M_{\odot}$, which leads us to model $\mathcal{D} \mathcal{S}_{\mathrm{M} 1}$. The FIMP scenario in $\mathcal{D} \mathcal{S}_{\mathrm{M} 1}$ makes almost the whole parameter set $\left\{g_{S}, m\right\}$ accessible, and hence one can always obtain $M_{\mathrm{d} D \mathcal{S}_{\mathrm{M} 1}} \sim 10^{10} M_{\odot}$. On the contrary, a dominant WIMP production mechanism fails to deliver the necessary values of $g_{S}$. Therefore, the missing satellite problem is easily solved for the FIMP case for sub-keV mediators, by considering for example $m=300 \mathrm{GeV}$, $m_{S}=0.1 \mathrm{keV}$ and $\alpha \approx 0.1$ or $m=1 \mathrm{TeV}, m_{S}=10 \mathrm{eV}$ and $\alpha \approx 1 / \pi$. All effective neutrino couplings are in perfect accordance with Ref. [10]. Alternatively, tuning $T_{\mathrm{c}}$, we assure that an instantaneous late kinetic decoupling at the desired temperature $T_{\mathrm{c}} \approx T_{\mathrm{kd}}$ takes place.

\section{Effects of SIDM cross sections on dwarf galaxies}

The final test of the dark matter models is given here: we investigate whether this parameter set supports noticeable effects on dwarf galaxies. As we mentioned before, the SIDM elastic cross section in galaxy clusters should lie within a constrained range of values. Quantitatively, the cross section for elastic scatterings between the dark matter particles should lie in the interval $\left\langle\sigma_{T} / m\right\rangle_{v_{\text {therm }}} \in[0.1,10] \mathrm{cm}^{2} \mathrm{~g}^{-1}$ [6] to resolve the cusp vs core problem as shown in Ref. [35], where the indicated average is taken with respect to a Maxwell-Boltzmann distribution with $v_{\text {therm }}=\mathcal{O}\left(10^{-4}\right)$ as the most probable velocity (or around $10^{-2}$ for massive clusters). For a detailed analysis see Ref. [36].

Exploring the parameter space of this family of models, we find that the values of SIDM elastic cross sections in 
model $\mathcal{D} \mathcal{S}_{\mathrm{M} 2}$ are of the desired order, $\sim 0.5-1 \mathrm{~cm}^{2} \mathrm{~g}^{-1}$ at dwarf scales. Therefore, after setting $x, g_{X}$ and $\ell$ to zero without loss of generality, no further modification of the dark sector is needed. However, sub-keV mediators need either $\mu$ dark matter charges or multi-TeV values of $m$ [16]. Both possibilities are strictly excluded, as found previously, due to the previous constraints, thermal evolution or perturbation theory. Therefore, in model $\mathcal{D} \mathcal{S}_{\mathrm{M} 1}$, as we mentioned before, we make use of the scalar potential, which should admit a phase transition at $T_{\mathrm{c}} \lesssim T_{\mathrm{kd}}$. This does not have an effect on the damping masses, but cures the SIDM values automatically. For the relevant subspace of parameters, the obtained values can stay always inside the constrained interval as one sees in Ref. [16]. The SIDM cross sections between $0.1-10 \mathrm{~cm}^{2} \mathrm{~g}^{-1}$ are easily accessible for values of dark matter resonances in the sub- $\mathrm{TeV} / \mathrm{TeV}$ range together with sub-GeV mediators. These results are in line with the maximum circular velocities observed and the values affecting dwarf galaxy scales [6]. For example, for the previous parameter set considering the FIMP scenarios with $m=0.3(1) \mathrm{TeV}$, a value of $M_{\mathrm{obs}}=$ $5(3) \mathrm{MeV}$ leads to the thermal averaged SIDM cross section values $\gtrsim 1.0 \mathrm{~cm}^{2} \mathrm{~g}^{-1}$ at the dwarf scales and $\lesssim 0.1 \mathrm{~cm}^{2} \mathrm{~g}^{-1}$ at cluster scales providing at the same time potential solutions to all enduring small structure problems of the $\Lambda$ CDM model, as stated in Ref. [36]. We stress that most of the parameter space from Fig. 3 in Ref. [37] is accessible in $\mathcal{D} \mathcal{S}_{\mathrm{M} 1}$ (FIMP scenario); for example, $m \approx 20 \mathrm{GeV}, \alpha \approx \alpha_{\mathrm{em}}$ and $m_{S} \approx 10 \mathrm{keV}$ lie inside the desired SIDM interval and provide $M_{\mathrm{d}} \approx 10^{10} M_{\odot}$. Finally, we note that after choosing the parameters to be $m \approx 1 \mathrm{TeV}, \alpha \approx 1 / \pi, m_{S} \approx 28 \mathrm{eV}$ and $M_{\mathrm{obs}} \approx 3 \mathrm{MeV}$, leading to $T_{\mathrm{kd}} \approx 420 \mathrm{eV}$, we are able to reproduce the ETHOS-4 model [2], which is cosmologically compatible and solves the missing satellite issue, the too-big-to-fail problem and the cusp vs core problem.

\section{THE UNDERLYING SPECTRUM}

In the present section we examine the resulting spectrum of the theory, which is able to provide solutions to the $\Lambda \mathrm{CDM}$ problems.

Besides the stable $F$, both the bosons and the sterile neutrino decay. In addition, for perturbative couplings $\bar{g}_{S} / 5=y=1 / 10$ and $g_{\nu}^{2}=10^{-13}$, the sterile neutrino admits a mass $M_{R} \approx 20 \mathrm{TeV}$ and $\delta r \approx 10^{-3}$. For this parameter set one finds that the $S$-singlet decouples from the SM-neutrino plasma at very high temperatures, around $m, M_{R}$, due to the tiny value of the effective neutrino coupling $g_{\nu}$; at that time almost all the d.o.f. of the standard model are relativistic. This means that $S, \Phi$ and $X$ freeze out early enough and there is not much time left for them to achieve local thermal equilibrium with neutrinos, which relaxes the strong constraints on $M_{\text {obs }}$ from Plank measurements [12]. This is a unique feature of this family of models, since the $S$ interactions at tree level include twopoint functions of $F$ 's or $n$ 's, which at the zero-momentum limit are severely suppressed due to the large masses, $m$ and $M_{R}$. Furthermore, the mass of $a$ is of order $m_{a} \sim \mu \mathrm{eV}$.

Recently, the IceCube Collaboration published results about extraterrestrial ultra-energetic neutrinos, i.e., Bert, Ernie [38] and Big Bird with approximate energies in the $\mathrm{PeV}$ regime. We could easily accommodate these observations in $\mathcal{D S} \mathcal{S}_{\mathrm{M} 1}: X$ admits a mass of $m_{X}=1 \mathrm{PeV}$ together with a minimum DM mass of $m=300 \mathrm{GeV}$; additional FIMP sources, i.e., more than one heavy $X$ boson, allow lower values of $m$ due to the constraints on the $g_{X}$ coupling (18). We stress that this parameter set still resolves all three sub-scale structure problems simultaneously after $T_{\mathrm{c}}$, delivering damping masses and SIDM cross sections of the desired order, together with the predicted value of $\Omega_{\mathrm{CDM}}$.

\section{CONCLUSION}

In this work we presented a proof of the existence of a purely phenomenological model, which resurrects the neutrinophilic theory solving the dark matter large- and small-scale problems, while respecting the experimental bounds and the standard model symmetries. To the best of our knowledge, there is no model which uses SM neutrinos as the last scattering partner for CDM. The proposed model admits only dimension-four operators, respects all cosmological and particle physics constraints and alleviates the small-structure problems of $\Lambda \mathrm{CDM}$ cosmology.

We introduced a singlet family of purely neutrinophilic dark matter models using SM neutrinos as dark radiation, thus proving that neutrinophilic theories are not over. The proposed dark sector interactions work as an extension of the SM of particle physics, while respecting the SM symmetries. We computed astrophysically important observables (cross sections and decay rates) in this framework and we presented and explained a list of constraints on the parameter space emerging from the particle physics and cosmological nature of the models. Finally, we estimated the chemical and kinetic decoupling and last scattering temperatures and we determined the relevant smallest protohalo masses. These masses are far above the Earth's mass produced by the usual WIMP models. The derived $M_{\mathrm{d}}$ alleviates the "missing satellite" problem and together with the SIDM cross sections provides a clear solution to the cuspy profile and massive subhalo issues of $\Lambda \mathrm{CDM}$ simultaneously.

In particular, the sterile neutrino works as the gaugeinvariant bridge between the standard model and the dark sector. It couples the SM-neutrino species to the stable dark matter candidate, $F$, with a strength compatible with particle physics constraints. Out-of-equilibrium decays of the heavy $X$ bosons achieve the required relic abundance of dark matter. The "missing satellite" problem is alleviated by the light singlet $S$ and the late decoupling properties of the SM neutrinos. The "cusp vs core" and the "too big to fail" 
issues are solved after the condensation of the scalar $\Phi$ happens.

It is worth noticing that in this model the strongly constrained $\mathrm{SU}(2)_{L}$ violating $\nu-S$ coupling arises naturally as an effective interaction. Furthermore, the case of a simple dominant WIMP production mechanism does not solve all the problems simultaneously and is ruled out, as we showed, at least in the perturbative regime of the coupling constant $\alpha$. Nevertheless, the FIMP scenario is able to alleviate all small-scale issues of the CDM paradigm. In addition, both dark matter production schemes provide excellent big bang nucleosynthesis/cosmic microwave background compatibility. Therefore, $\Lambda \mathrm{CDM}$ models are still viable and seem more attractive in this neutrinophilic perspective.
The straightforwardness of this phenomenological model is intriguing. In future works we hope to return to this model, investigate the possibility of a more minimal construction and consider different spectrum hierarchies.

\section{ACKNOWLEDGMENTS}

It is a great pleasure to thank Frederik Lauf and Kerstin Paech for inspiring discussions. We are grateful for comments by Torsten Bringmann who helped to clarify the presentation of our work. We appreciate financial support of our work from the Deutsche Forschungsgemeinschaft (DFG) cluster of excellence "Origin and Structure of the Universe," the Humboldt Foundation, and from Transregio 33 (TRR 33) "The Dark Universe." O. B. is grateful for financial support from the "FAZIT-STIFTUNG."
[1] S. Dodelson and L. M. Widrow, Sterile-Neutrinos as Dark Matter, Phys. Rev. Lett. 72, 17 (1994).

[2] M. Vogelsberger, J. Zavala, F.-Y. Cyr-Racine, C. Pfrommer, T. Bringmann, and K. Sigurdson, ETHOS-An effective theory of structure formation: Dark matter physics as a possible explanation of the small-scale CDM problems, Mon. Not. R. Astron. Soc. 460, 1399 (2016).

[3] T. Bringmann, H. T. Ihle, J. Kersten, and P. Walia, Suppressing structure formation at dwarf galaxy scales and below: Late kinetic decoupling as a compelling alternative to warm dark matter, Phys. Rev. D 94, 103529 (2016).

[4] P. J. Fox and E. Poppitz, Leptophilic dark matter, Phys. Rev. D 79, 083528 (2009).

[5] I. M. Shoemaker, Constraints on dark matter protohalos in effective theories, and neutrinophilic dark matter, Phys. Dark Universe 2, 157 (2013).

[6] T. Bringmann, F. Kahlhoefer, K. Schmidt-Hoberg, and P. Walia, Strong Constraints on Self-Interacting Dark Matter with Light Mediators, Phys. Rev. Lett. 118, 141802 (2017).

[7] L. G. van den Aarssen, T. Bringmann, and Y. C Goedecke, Thermal decoupling and the smallest subhalo mass in dark matter models with Sommerfeld-enhanced annihilation rates, Phys. Rev. D 85, 123512 (2012).

[8] R. Laha, B. Dasgupta, and J. F. Beacom, Constraints on new neutrino interactions via light Abelian vector bosons, Phys. Rev. D 89, 093025 (2014).

[9] P. S. Pasquini and O. L. G. Peres, Bounds on neutrino-scalar Yukawa coupling, Phys. Rev. D 93, 053007 (2016); Erratum 93, 079902 (2016).

[10] L. Heurtier and Y. Zhang, Supernova constraints on massive (pseudo)scalar coupling to neutrinos, J. Cosmol. Astropart. Phys. 02 (2017) 042.

[11] R. Foot and S. Vagnozzi, Dissipative hidden sector dark matter, Phys. Rev. D 91, 023512 (2015).

[12] J.H. Heo and C.S. Kim, Light dark matter and dark radiation, J. Korean Phys. Soc. 68, 715 (2016).
[13] P. S. B. Dev, C. M. Vila, and W. Rodejohann, Naturalness in testable type II seesaw scenarios, Nucl. Phys. B921, 436 (2017).

[14] J. Schechter and J. W. F. Valle, Neutrino masses in $\mathrm{SU}(2) \times$ U(1) theories, Phys. Rev. D 22, 2227 (1980).

[15] M. E. Carrington, The effective potential at finite temperature in the Standard Model, Phys. Rev. D 45, 2933 (1992).

[16] S. Tulin, H.-B. Yu, and K. M. Zurek, Beyond collisionless dark matter: Particle physics dynamics for dark matter halo structure, Phys. Rev. D 87, 115007 (2013).

[17] K. Griest and M. Kamionkowski, Unitarity Limits on the Mass and Radius of Dark Matter Particles, Phys. Rev. Lett. 64, 615 (1990).

[18] C. Patrignani et al., Review of particle physics, Chin. Phys. C 40, 100001 (2016).

[19] A. V. Artamonov et al., Search for the rare decay $K^{+} \rightarrow \mu^{+} \nu \bar{\nu} \nu$, Phys. Rev. D 94, 032012 (2016).

[20] R. Abbasi et al., Search for neutrinos from annihilating dark matter in the direction of the Galactic center with the 40-string IceCube Neutrino Observatory, arXiv:1210.3557.

[21] K. Enqvist, K. Kainulainen, and V. Semikoz, Neutrino annihilation in hot plasma, Nucl. Phys. B374, 392 (1992).

[22] R. H. Cyburt, B. D. Fields, K. A. Olive, and T.-H. Yeh, Big bang nucleosynthesis: 2015, Rev. Mod. Phys. 88, 015004 (2016).

[23] P. A. R. Ade et al., Planck 2015 results. XIII. Cosmological parameters, Astron. Astrophys. 594, A13 (2016).

[24] J. Baur, N. Palanque-Delabrouille, C. Yche, C. Magneville, and M. Viel, Lyman-alpha forests cool warm dark matter, J. Cosmol. Astropart. Phys. 08 (2016) 012.

[25] V. Iršič et al., New Constraints on the free-streaming of warm dark matter from intermediate and small scale Lyman$\alpha$ forest data, Phys. Rev. D 96, 023522 (2017).

[26] A. Garzilli, A. Boyarsky, and O. Ruchayskiy, Cutoff in the Lyman $\alpha$ forest power spectrum: Warm IGM or warm dark matter? Phys. Lett. B 773, 258 (2017). 
[27] T. Bringmann and S. Hofmann, Thermal decoupling of WIMPs from first principles, J. Cosmol. Astropart. Phys. 04 (2007) 016; Erratum, 03 (2016) E02.

[28] P. Gondolo and K. Kadota, Late kinetic decoupling of light magnetic dipole dark matter, J. Cosmol. Astropart. Phys. 06 (2016) 012.

[29] A. Loeb and M. Zaldarriaga, The small-scale power spectrum of cold dark matter, Phys. Rev. D 71, 103520 (2005).

[30] A. M. Green, S. Hofmann, and D. J. Schwarz, The first WIMPy halos, J. Cosmol. Astropart. Phys. 08 (2005) 003.

[31] L. G. van den Aarssen, T. Bringmann, and C. Pfrommer, Is dark matter with long-range interactions a solution to all small-scale problems of $\Lambda$ CDM cosmology? Phys. Rev. Lett. 109, 231301 (2012).

[32] J.S. Bullock, Notes on the missing satellites problem, arXiv:1009.4505.
[33] E. Piceno, A. Rosado, and E. Sadurn, Fundamental constraints on two-time physics, Eur. Phys. J. Plus 131, 352 (2016).

[34] S. Hofmann, D. J. Schwarz, and H. Stoecker, Damping scales of neutralino cold dark matter, Phys. Rev. D 64, 083507 (2001).

[35] J. Zavala, M. Vogelsberger, and M. G. Walker, Constraining self-interacting dark matter with the Milky Way's dwarf spheroidals, Mon. Not. R. Astron. Soc. 431, L20 (2013).

[36] S. Tulin and H.-B. Yu, Dark matter self-interactions and small scale structure, Phys. Rep., 730, 1 (2018).

[37] M. Kaplinghat, S. Tulin, and H.-B. Yu, Dark Matter Halos as Particle Colliders: Unified Solution to Small-Scale Structure Puzzles from Dwarfs to Clusters, Phys. Rev. Lett. 116, 041302 (2016).

[38] M. G. Aartsen et al., First Observation of Pev-Energy Neutrinos with IceCube, Phys. Rev. Lett. 111, 021103 (2013). 